\section{(A) Check for updates}

Cite this: Polym. Chem., 2020, 11 5861

Received 24th June 2020 Accepted 18th August 2020

DOI: $10.1039 /$ d0py00912a

rsc.li/polymers

\title{
An improved synthesis of poly(amidoamine)s for complexation with self-amplifying RNA and effective transfection $\uparrow$
}

\author{
Pratik Gurnani, (D)*a Anna K. Blakney, (DD ${ }^{b}$ Jonathan Yeow, (D) Clément R. Bouton, ${ }^{\mathrm{b}}$ \\ Robin J. Shattock, ${ }^{\mathrm{b}}$ Molly M. Stevens (10) ${ }^{\mathrm{c}}$ and Cameron Alexander (1D*a
}

\begin{abstract}
Cationic polymers are widely used as materials to condense nucleic acids for gene-based therapies. These have been developed to mainly deliver DNA and RNA for cancer therapies but the ongoing COVID-19 pandemic has demonstrated an urgent need for new DNA and RNA vaccines. Given this, suitable manufacturing conditions for such cationic polymers which can protect the nucleic acid in the formulation and delivery stages but release the cargo in the correct cellular compartment effectively and safely are required. A number of polymers based on poly(amidoamine)s fit these criteria but their syntheses can be time-consuming, inefficient and poorly reproducible, precluding their adoption as manufacturable vaccine excipients. Here we report an improved synthesis of poly(cystamine bisacrylamide-co-4amino-1-butanol), abbreviated as $\mathrm{pABOL}$, via modifications in concentration, reaction time and reaction conditions. Optimisation of monomer contents and stoichiometries, solvents, diluents and temperature, combined with the application of microwaves, enabled the preparation of vaccine candidate $\mathrm{pABOL}$ materials in $4 \mathrm{~h}$ compared to $48 \mathrm{~h}$ reported for previous syntheses. These procedures were highly reproducible in multiple repeat syntheses. Transfection experiments with a model RNA showed that polymers of formulation with appropriate molar masses and mass distributions were as effective in model cell lines as polymers derived from the unoptimised syntheses which have been shown to have high efficacy as RNA vaccine formulation candidates.
\end{abstract}

\section{Introduction}

The application of polycations in the formulation of nucleic acid delivery systems has accelerated rapidly in recent years. ${ }^{1-4}$ Advances in gene editing technologies, and the possibilities for DNA- and RNA-based therapies and vaccines against cancers and infectious diseases, have all contributed to the focus on polycation-based gene delivery formulations. ${ }^{5-9}$ Within the large field of cationic polymers, materials based on oligo- or poly(amidoamine)s (pAMAMs) and poly ( $\beta$-aminoesters) (pBAEs) have been extensively investigated. ${ }^{10-16}$ This is because these polymers are intrinsi-

\footnotetext{
${ }^{a}$ Division of Molecular Therapeutics and Formulation, School of Pharmacy, University of Nottingham, NG7 2RD, UK. E-mail: pratik.gurnani@nottingham.ac.uk, cameron.alexander@nottingham.ac.uk

${ }^{b}$ Department of Infectious Disease, Imperial College London, School of Medicine, St Mary's Hospital, Praed Street, London W2 1NY, UK

${ }^{c}$ Department of Materials and the Department of Bioengineering at Imperial College London, Prince Consort Rd, SW7 2AZ London, South Kensington, UK

$\dagger$ Electronic supplementary information (ESI) available. See DOI: 10.1039/ d0py00912a
}

cally biodegradable and can be synthesised from a range of commercially available bisacrylamide/bisacrylates (for pAMAMs/pBAEs respectively) and primary amines, thus enabling high chemical diversity and consequent broad utility across different nucleic acid delivery platforms. ${ }^{17}$ pAMAMs in particular have shown particular promise due to their limited mid-chain hydrolysis and higher water solubility due to their amide backbone, in comparison to esters in pBAEs. However, while the preparation of pAMAMs is relatively straight-forward for research investigations, ${ }^{18}$ in the case of rapid manufacture potentially required for high volume nucleic acid vaccine production, the current synthetic protocols are sub-optimal. In particular, there is a pressing need to understand if methods used for the preparation of PAMAMs can be tuned to produce materials with the right properties for delivery of mRNA or other nucleic acid vaccine candidates in a reproducible way, and in a manner which might allow for rapid and distributed manufacture.

Accordingly, the aim of the study presented here was to investigate the synthetic parameters leading to reproducible and faster synthesis of a specific class of pAMAMs, whilst potentially making the procedure more amenable to micro- 
wave acceleration and continuous flow technologies for scale up and potential decentralised manufacture. The specific focus was to develop a more rapid synthesis of poly(cystamine bisacrylamide-co-4-amino-1-butanol), hereinafter referred to as pABOL, which has shown promise as a highly efficient delivery vector for self-amplifying RNA (saRNA) in candidate vaccine formulations. ${ }^{19}$ Pioneering work on this polymer, ${ }^{20}$ and related materials, ${ }^{21-24}$ initially established broad efficacy across a range of gene delivery applications, with the bioreducible disulfide linker greatly enhancing the expression of transgenes and minimising vector toxicity. ${ }^{25}$ In most reports, the synthesis of pABOL is conducted via the step-growth Michaeladdition polymerisation between $N, N^{\prime}$-cystaminebisacrylamide and 4-amino-1-butanol, either without solvent, or in high concentrations in apolar solvents. These methods typically allow the generation of polymers with molar masses of $10^{3}-10^{4} \mathrm{~g}$ $\mathrm{mol}^{-1}$ and $D$ values above 2 , but reactions under these conditions require several days to reach high molar masses. The demand for sufficient cationic polymer for many millions of vaccine doses to be made rapidly available is incompatible with reactions which take long time periods. There is now a requirement to develop methods by which pABOL-type polymers can be produced to an appropriate specification for reproducible vaccine formulations in a minimum time frame. Accelerated polymerisations, may also be relevant in structureproperty optimisation studies to screen structural (molar mass, branching etc.) and chemical (lipophilicity, basicity, charge density etc.). However, the current slow synthetic methods for this family of polymers significantly hinders structure optimisation, potentially useful for tailoring polymer vectors to an emerging nucleic acid vaccine candidate.

We report here a synthetic optimisation study, screening the effect of reaction parameters such as inert atmospheres, monomer concentration, reactant ratio and reaction temperature on the molecular weight distribution of pABOL. Through these improvements, we were able to minimise a radical branching side reaction, then accelerate the prevailing stepgrowth polymerisation with microwave assisted synthesis. The conditions established in this work were highly reproducible in comparison to unoptimized conditions. Finally, we highlight the importance of controlling the molecular weight distribution by evaluating the correlation of certain polymer physicochemical characteristics and their propensity to effectively deliver a model saRNA vaccine.

\section{Experimental}

\section{Materials}

All solvents were of analytic or HPLC grade and purchased from Sigma Aldrich or Fisher Scientific unless otherwise stated. All deuterated solvents were purchased from Sigma Aldrich. Reagents 4-aminobutanol (178330-5G), N,N'-bis(acryloyl)cystamine (A4929-5G) and triethylamine were purchased from Sigma-Aldrich. fLuc VEEV saRNA (9382 nt) was prepared as described previously. ${ }^{26}$

\section{Instrumentation}

${ }^{1} \mathrm{H}$ NMR spectroscopy. ${ }^{1} \mathrm{H}$ NMR, spectra were recorded at $25{ }^{\circ} \mathrm{C}$ on a Bruker Advance III $400 \mathrm{MHz}$ spectrometer. All chemical shifts are reported in $\operatorname{ppm}(\delta)$ referenced to the chemical shifts of residual solvent resonances of $d_{6}$-DMSO (2.50 ppm). MestReNova 6.0.2 copyright 2009 (Mestrelab Research S. L.) was used for analysing the spectra.

Size exclusion chromatography. A Polymer Laboratories PL-50 instrument equipped with differential refractive index (DRI), viscometry (VS) and dual angle light scatter (LS) was used for SEC analysis. The system was fitted with $2 \times$ PLgel Mixed D columns $(300 \times 7.5 \mathrm{~mm})$ and a PLgel $5 \mu \mathrm{m}$ guard column. The eluent used was DMF with $0.1 \%$ LiBr. Samples were run at $1 \mathrm{ml} \mathrm{min}^{-1}$ at $50{ }^{\circ} \mathrm{C}$. Poly(methyl methacrylate) standards (Agilent EasyVials) were used for calibration between 955 500-550 $\mathrm{g} \mathrm{mol}^{-1}$. Analyte samples were filtered through a membrane with $0.22 \mu \mathrm{m}$ pore size before injection. Respectively, experimental molar mass $\left(M_{\mathrm{n}, \mathrm{SEC}}\right)$ and dispersity $(\nexists)$ values of synthesized polymers were determined by conventional calibration using Cirrus GPC software.

\section{Polymer synthesis}

General procedure for synthesis of pABOL. pABOL was synthesised via the following general procedure, the unoptimised procedure is detailed here. In a typical experiment, CBA, ABOL and triethylamine were added into a test tube charged with a stir bar and the reagents suspended in a $\mathrm{MeOH} /$ water mixture $(4 / 1, v / v)$ and the tube sealed with a rubber septum. Polymerization was carried out in the dark at $45{ }^{\circ} \mathrm{C}$ under a static nitrogen atmosphere. Polymers utilised in transfection studies were isolated using the following procedure: the resulting solution was diluted with $\mathrm{MeOH}(50 \mathrm{~mL})$, acidified with 1 $\mathrm{M} \mathrm{HCl}$ to $\mathrm{pH} \sim 4$, and then purified by dialysis with acidic water ( $4.0 \mathrm{~L}, \mathrm{pH} \sim 5$, refreshed 6 times in 3 days). The polymers in their $\mathrm{HCl}$-salt form were collected as white solid after lyophilisation.

\section{Formulation of saRNA polyplexes}

Stock solutions of pABOLs and saRNA were prepared first by directly dissolving these materials in molecular grade water and stored at $4{ }^{\circ} \mathrm{C}$ for up to 3 months. The concentrations of the stock solutions are $0.24 \mu \mathrm{g} \mu \mathrm{L}^{-1}$ (fLuc VEEV saRNA), and $5.00 \mu \mathrm{g} \mu \mathrm{L}^{-1}$ (pABOLs) respectively.

\section{In vitro transfection of saRNA polyplexes}

Transfections were performed in HEK293T.17 cells (ATCC, USA) that were maintained in culture in complete Dulbecco's Modified Eagle's Medium (cDMEM) (Gibco, ThermoFisher, UK) containing $10 \%(\mathrm{v} / \mathrm{v})$ fetal calf serum (FCS), $5 \mathrm{mg} \mathrm{mL}$ L-glutamine and $5 \mathrm{mg} \mathrm{mL} \mathrm{mL}^{-1}$ penicillin and streptomycin (ThermoFisher, UK). Cells were plated at a density of 50000 cells per well in a 96 well plate $24 \mathrm{~h}$ prior to transfection. At the time of transfection, the media was completely removed and replaced by $50 \mu \mathrm{L}$ of transfection media (DMEM with $5 \mathrm{mg} \mathrm{mL}{ }^{-1}$ L-glutamine). $100 \mu \mathrm{L}$ of the polyplex solution was 
added to each well and incubated for $4 \mathrm{~h}$. Then, the media was replaced with cDMEM and the cells were allowed to culture for 24 hours, at which time $50 \mu \mathrm{L}$ of media was removed and $50 \mu \mathrm{L}$ of ONE-Glo ${ }^{\mathrm{TM}}$ D-luciferin substrate (Promega, UK) was added and mixed well by pipetting. The total volume was transferred to a white 96-well plate (Costar, UK) and analyzed on a FLUOstar Omega plate reader (BMG LABTECH, UK).

\section{Results and discussion}

Previous reports of the synthesis of pABOL have utilised protocols where $N, N^{\prime}$-bis(acryloyl)cystamine (CBA), 4-aminobutanol (ABOL) and triethylamine in a methanol/water are mixed and heated with stirring at $45{ }^{\circ} \mathrm{C}$ under a nitrogen atmosphere for up to 14 days. ${ }^{19}$ We thus commenced our studies by following this synthesis (Fig. 1) and observed a clear viscous solution at the end of two weeks heating at $45{ }^{\circ} \mathrm{C}$ (Table 1 , entry 1). NMR spectra (Fig. 1B) and DMF-SEC revealed successful polymerisation, with an $M_{\mathrm{w}, \mathrm{SEC}}=45 \mathrm{~kg} \mathrm{~mol}^{-1}$ and a relatively broad molecular weight distribution $(\nexists=3.62)$. The origins of this high $D$ appeared to be due to an additional high molecular weight peak in the chromatogram, which was postulated to be caused by chain branching through a side reaction of radical polymerisation (Fig. 1C).

These results suggest that the high reaction concentration (3.2 M) and inert conditions both favour this side reaction. We thus conducted a systematic study, varying the reaction conditions to mitigate this side reaction. Previous data indicated that pABOL of $M_{\mathrm{w}} \sim 8 \mathrm{~kg} \mathrm{~mol}^{-1}$ yields high saRNA transfection in animal models. Therefore, throughout the optimisation study we targeted polymers around this molecular weight.

\section{Systematic optimisation of polymerisation}

Effect of dilution and inert conditions. Radical polymerisations are favoured under inert conditions (oxygen is known to act as a radical scavenger), further exacerbated at high monomer concentrations. ${ }^{27,28}$ We therefore evaluated the molecular weight evolution over 2 weeks for the step-growth polymerisation at a $\sim 5$-fold dilution $(0.6 \mathrm{M}$ instead of $3.2 \mathrm{M}$ ) under inert (argon deoxygenated) compared to a polymerisation under air (oxygenated) to limit the radical polymerisation side reaction.

Immediately comparing the argon deoxygenated conditions at lower dilution (Table 1, entry 2), we observed a steady increase in molar mass without the appearance of a bimodal high molar mass peak. A high molar mass shoulder however, was observed after $10 \mathrm{~d}$ in the SEC chromatograms, indicating some radical polymerisation was still occurring at diluted deoxygenated conditions (Fig. 2A). This was further evidenced by the minimal increase in $M_{\mathrm{n}, \mathrm{SEC}}$ whilst $M_{\mathrm{w}}$,SEC continued to climb after this time point. Noticeably, the diluted conditions appear to retard the side reaction, however, do not completely

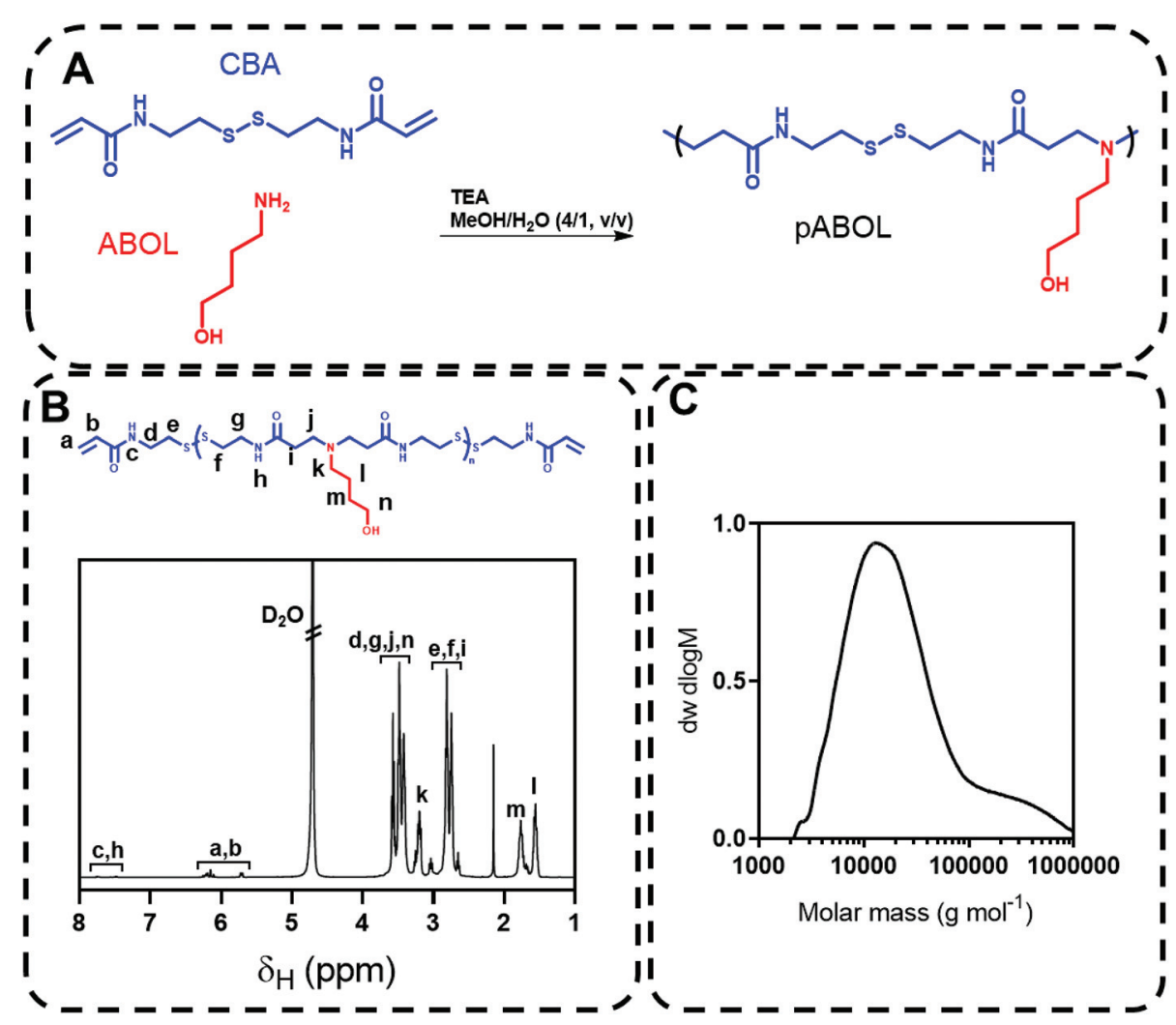

Fig. 1 (A) Synthetic scheme for step-growth Michael-addition polymerisation of $N, N^{\prime}$-cystaminebisacrylamide and 4-amino-1-butanol to produce pABOL, (B) ${ }^{1} \mathrm{H}$ NMR spectrum of $\mathrm{pABOL}$; (C) size exclusion chromatogram of product obtained via 'conventional' synthesis. 
Table 1 Polymerisation conditions for pABOL syntheses reported in this study

\begin{tabular}{|c|c|c|c|c|c|c|}
\hline Description & Entry & {$[\mathrm{ABOL}](\mathrm{M})$} & {$[\mathrm{CBA}](\mathrm{M})$} & {$[\mathrm{ABOL}] /[\mathrm{CBA}]$} & Atmosphere & $T\left({ }^{\circ} \mathrm{C}\right)$ \\
\hline Original & 1 & 3.2 & 3.2 & 1 & Argon & 45 \\
\hline Dilution & 2 & 0.60 & 0.60 & 1 & Argon & 45 \\
\hline Dilution + air & 3 & 0.60 & 0.60 & 1 & Air & 45 \\
\hline \multirow[t]{2}{*}{ Temperature } & 4 & 0.60 & 0.60 & 1 & Air & 60 \\
\hline & 5 & 0.60 & 0.60 & 1 & Air & 25 \\
\hline \multirow[t]{4}{*}[\mathrm{M}]{} & 6 & 3.2 & 3.2 & 1 & Air & 60 \\
\hline & 7 & 0.8 & 0.8 & 1 & Air & 60 \\
\hline & 8 & 0.45 & 0.45 & 1 & Air & 60 \\
\hline & 9 & 0.24 & 0.24 & 1 & Air & 60 \\
\hline \multirow[t]{6}{*}{ Monomer ratio } & 10 & 0.60 & 0.60 & 1 & Air & 60 \\
\hline & 11 & 0.606 & 0.60 & 1.01 & Air & 60 \\
\hline & 12 & 0.615 & 0.60 & 1.025 & Air & 60 \\
\hline & 13 & 0.63 & 0.60 & 1.05 & Air & 60 \\
\hline & 14 & 0.66 & 0.60 & 1.1 & Air & 60 \\
\hline & 15 & 0.72 & 0.60 & 1.2 & Air & 60 \\
\hline \multirow[t]{2}{*}{ Repeatability } & 16 & 0.60 & 0.60 & 1 & Air & 60 \\
\hline & 17 & 3.2 & 3.2 & 1 & Argon & 45 \\
\hline Microwave & 18 & 0.60 & 0.60 & 1 & Air & 100 \\
\hline
\end{tabular}
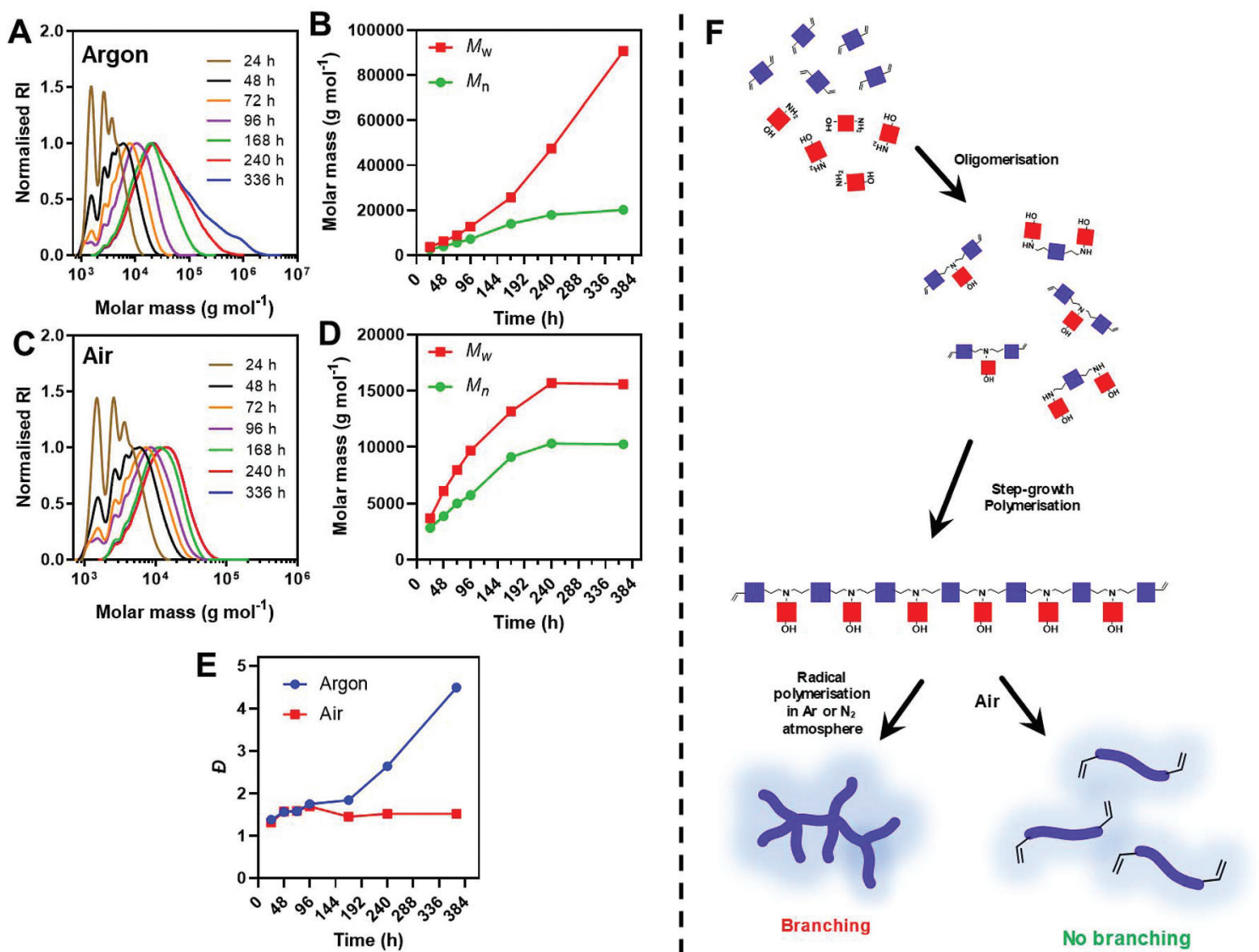

Fig. 2 (A and C) DMF-SEC chromatograms and (B and D) molecular weight evolution of pABOL at $[\mathrm{M}]=0.60 \mathrm{M}, T=45^{\circ} \mathrm{C}$ polymerisation in an argon or air atmosphere. (E) Evolution of dispersity over time for PABOL. (F) Schematic representation of step-growth polymerisation and subsequent radical polymerisation leading to branching under inert conditions and inhibition of radical polymerisation under air. 
inhibit it. Comparatively at the $10 \mathrm{~d}$ time point under air (Table 1, entry 3), no further increase in molecular weight was observed, likely due to the radical scavenging mechanism of oxygen (Fig. 2B). This is further exemplified in the $D$ values, with samples taken from polymerisations performed under air maintaining $D<1.6$, while $D$ for polymers prepared under inert atmosphere rose to $\sim 4.5$ over the 2 weeks reaction (Fig. 2C).

We anticipate that the steadily increasing high molar mass shoulder was a result of successive branching events, otherwise inhibited under an air atmosphere (Fig. 2D). The diluted conditions significantly slowed the rate of polymerisations taking $96 \mathrm{~h}$ to reach $M_{\mathrm{w}, \mathrm{SEC}}>8 \mathrm{~kg} \mathrm{~mol}^{-1}$, whilst concentrated conditions (5-fold higher concentration), reached these molar masses within $48 \mathrm{~h}$.

Effect of temperature and microwave acceleration. One of the other parameters which may affect the rate of this branching mechanism, even under oxygenated conditions, is temperature. We compared polymerisations performed at $60{ }^{\circ} \mathrm{C}$ (Table 1, entry 4) (close to the boiling point of methanol solvent) and $25^{\circ} \mathrm{C}$ (Table 1 , entry 5), compared to the previous results obtained at $45^{\circ} \mathrm{C}$ (Table 1 , entry 3 ), monitoring molecular weight evolution daily for $96 \mathrm{~h}$. As expected, we observed significantly faster growth in molecular weight at the higher temperatures, with reactions performed at $60^{\circ} \mathrm{C}$ plateauing within $48 \mathrm{~h}$ at $M_{\mathrm{w}} \sim 10 \mathrm{~kg} \mathrm{~mol}^{-1}$. Lower temperatures slowed the polymerisation significantly, only reaching $\sim 3 \mathrm{~kg} \mathrm{~mol}^{-1}$ within 96 h (Fig. 3A and C). Notably, although still under a higher dilution, polymerisations at $60{ }^{\circ} \mathrm{C}$ reached the target $8 \mathrm{~kg} \mathrm{~mol}{ }^{-1}$ within $48 \mathrm{~h}$, the same timeframe as the concentrated conditions at $45{ }^{\circ} \mathrm{C}$. Importantly no evidence of branch- ing was observed even at the $96 \mathrm{~h}$ timepoint, $2 \mathrm{~d}$ after the polymerisation terminated. Overall, higher temperatures did not induce more branching, but did increase the rate of polymerisation sufficiently to compensate for the diluted conditions.

With these conditions providing low viscosity solutions and exhibiting minimal side reactions we sought to accelerate further the synthetic procedure utilising microwave irradiation. Microwave accelerated synthesis has two major advantages: firstly, microwaves homogenously heat solvents, leading to reliable heat gradients; secondly most microwave synthesis procedures occur under high pressure conditions, increasing the boiling point of solvent. ${ }^{29,30}$ These effects both synergise and enable synthesis at significantly higher temperatures and faster reaction rates than conventional heating. Furthermore, microwave accelerated synthesis is highly amenable to continuous flow reactors as they offer fast, but energy efficient synthetic routes to scalable manufacture, important in rapid response supply chains. ${ }^{31}$

To evaluate $\mathrm{pABOL}$ synthesis using microwave assisted heating, we utilised a Biotage microwave synthesiser set to 200 $\mathrm{W}$, heating to $100{ }^{\circ} \mathrm{C}$ and monitored the molecular weight evolution over $24 \mathrm{~h}$ (Table 1, entry 18). Under these conditions, we observed a significantly faster increase in molecular weight, reaching $\sim 8 \mathrm{kDa}$ in $\sim 4 \mathrm{~h}$ rather than $48 \mathrm{~h}$, a 12-fold acceleration compared to conventional heating at $60{ }^{\circ} \mathrm{C}$ (Fig. $3 \mathrm{~B}$ and C). Chromatograms were comparable under microwave conditions and conventional, with similar $Ð$ regardless of heating method.

Effect of monomer concentration and ratio. Having previously observed that the polymerisation plateaus without branching under oxygenated conditions, we postulated that
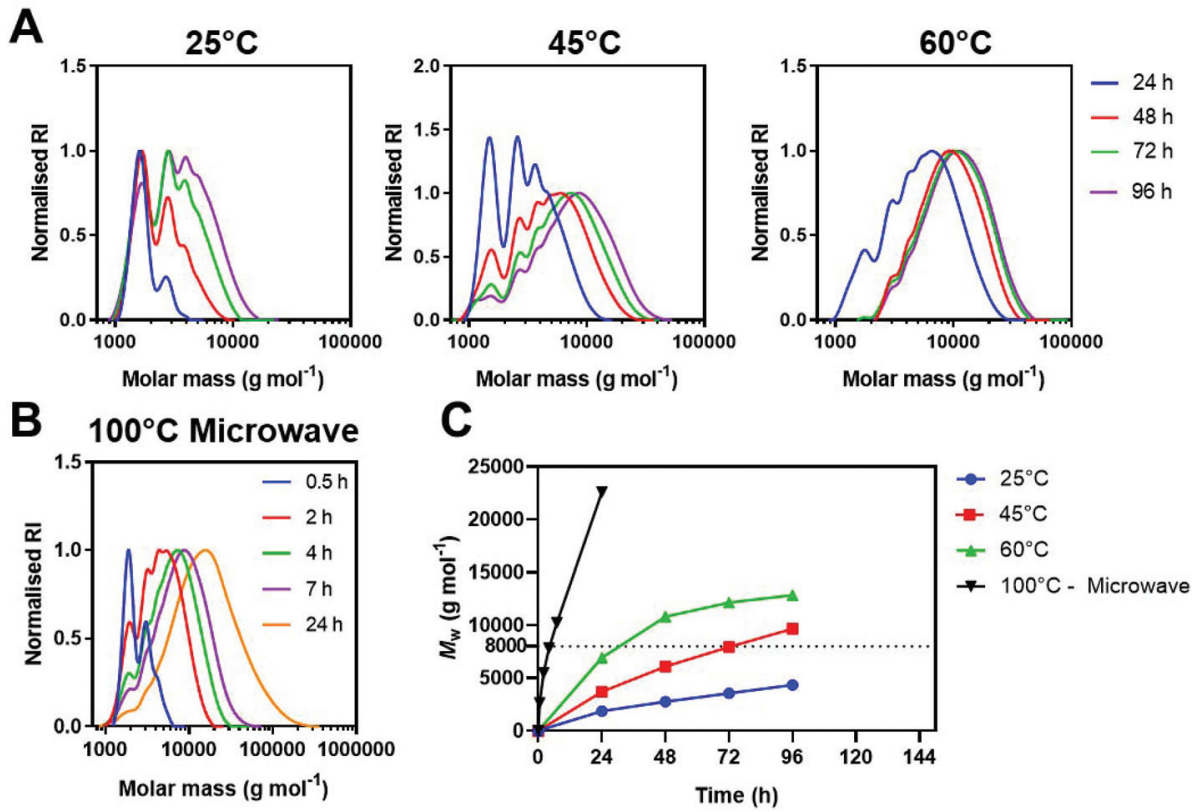

Fig. 3 (A and B) DMF-SEC chromatograms and (C) molecular weight evolution of $\mathrm{PABOL}$ at $[\mathrm{M}]=0.60 \mathrm{M}$ and air atmosphere at $25^{\circ} \mathrm{C}, 45^{\circ} \mathrm{C}$ and $60^{\circ} \mathrm{C}$ under conventional heating at $100^{\circ} \mathrm{C}$ under using microwave assisted heating. 
the molecular weight at which this termination takes place may be controlled in a concentration dependent manner. It is well established that as step-growth polymerisations ensue, higher concentrations help overcome steric effects which limit chain-chain addition steps. ${ }^{32}$ To probe this, we performed polymerisations at 4 concentrations and monitored the evolution of molecular weight daily over $72 \mathrm{~h}$. A solution of the original conditions was aliquotted equally into 4 tubes, and each diluted with the methanol $/ \mathrm{H}_{2} \mathrm{O}$ mixture such that they were at 3.2 $\mathrm{M}$ (no extra solvent), $0.8 \mathrm{M}, 0.45 \mathrm{M}$ and $0.24 \mathrm{M}$ (Table 1, entries 6-9) and heated to $60^{\circ} \mathrm{C}$ under air. For the lower three concentrations we observed that the polymerisation terminated after $48 \mathrm{~h}$, but achieving lower molecular weights for lower polymerisation concentrations (Fig. 4A and $\mathrm{B}$ ). At the highest concentration, we observed that after $72 \mathrm{~h}$, a high molar mass shoulder appeared potentially indicating some branching may occur even at high concentrations in an air atmosphere.

To achieve high molar masses for step-growth polymerisations it is imperative to have a near equimolar ratio between the $\mathrm{A}$ and $\mathrm{B}$ components. However, if the ratios are not perfectly equimolar (i.e. $1-10 \%$ out), it is possible to tune the molecular weight and end-group of the final polymers. We prepared 5 polymerisations at an equimolar ratio and added $1 \%$, $2.5 \%, 5 \%, 10 \%$ and $20 \%$ (Table 1 , entries 11-15) excess ABOL and heated the tubes for $48 \mathrm{~h}$ at $60{ }^{\circ} \mathrm{C}$ under an air atmosphere. With $1 \%$ extra ABOL we observed no difference in final $M_{\mathrm{w}}$, compared to the purely equimolar polymerisation, however with higher amounts of excess ABOL a decreasing trend in $M_{\mathrm{w}}$ with increasing excess ABOL was evident (Fig. 4C and D). At $20 \%$ excess ABOL, no polymerisation or only oligomerisation was observed. This data also provides important information on the weighing/stoichiometric error allowed (max. 1\%) to avoid molecular weight discrepancy.

Repeatability. The optimised conditions $([\mathrm{M}]=0.60 \mathrm{M}$, $T=60{ }^{\circ} \mathrm{C}$, air atmosphere) yielded a low viscosity solution, with minimal branching observed in SEC chromatograms. We therefore studied the repeatability of the conditions, preparing 3 independent reactions for the optimised (Table 1, $3 \times$ entry 16) and original conditions (Table $1,3 \times$ entry 17). After $48 \mathrm{~h}$ at $60{ }^{\circ} \mathrm{C}$ under an air atmosphere, the polymerisations were stopped and the molecular weight measured via DMF-SEC. The three polymerisations performed under the 'new' conditions had almost identical chromatograms, with an average $M_{\mathrm{w}}=9100 \pm 610 \mathrm{~g} \mathrm{~mol}^{-1}$ and $Ð=1.31 \pm 0.00$. In contrast, polymerisations with the 'old' conditions exhibited an average $M_{\mathrm{w}}=16700 \pm 5500 \mathrm{~g} \mathrm{~mol}^{-1}$ and $D=3.51 \pm 1.07$ (Fig. $5 \mathrm{~A}-\mathrm{C}$ ). The variation in $M_{\mathrm{w}}$ and $D$ for the 'old' conditions is most likely a consequence of the bimodal distribution, absent in the 'new' conditions. Overall, the new conditions presented here generated polymers with more reproducible properties.

Effect of polymer properties on biological activity. In view of the potential applications of pABOL in gene delivery, ${ }^{19}$ and specifically in the need for rapid deployment as components in nucleic acid vaccine formulations, we evaluated pABOLs, P1-P6, synthesised by a range of conditions for efficacy in transfection (Fig. 6A and B). These experiments were carried out to assess how the spread of molar masses, polydispersities and other chromatogram features (e.g. unimodal vs. bimodal molecular weight distributions) of polymers made under
A
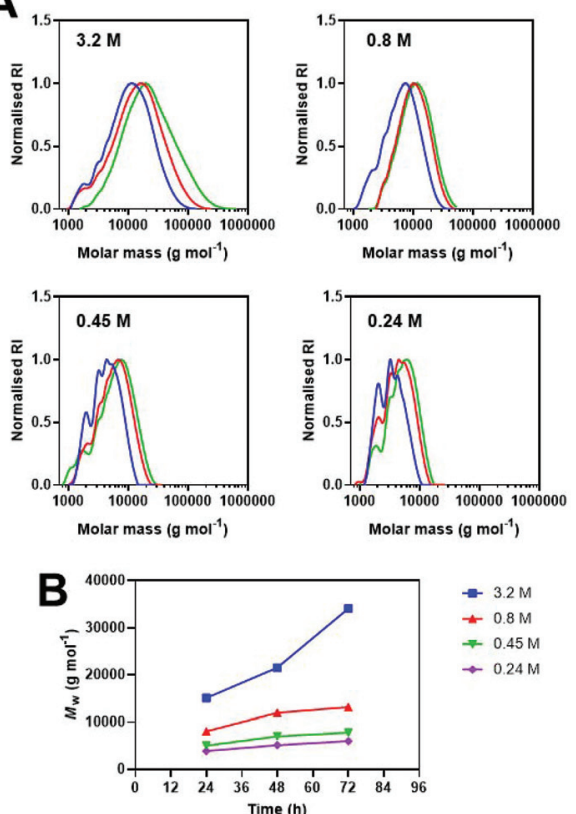

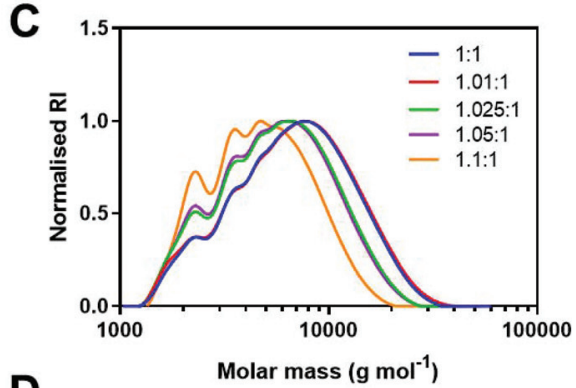

D

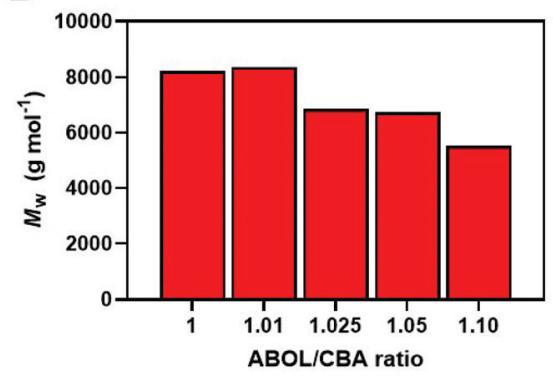

Fig. 4 (A) DMF-SEC chromatograms and (B) molecular weight evolution of $A B O L+C B A$ polymerisations at $60^{\circ} \mathrm{C}$ and an air atmosphere at $[M]=$ 3.2, 0.8, 0.45 and 0.24 M. (C) DMF-SEC chromatograms and (D) $M_{\mathrm{w}, \mathrm{SEC}}$ of final polymers after $48 \mathrm{~h}$ reaction between CBA and ABOL at different $\mathrm{ABOL} / \mathrm{CBA}$ ratios. Reactions were performed at $60^{\circ} \mathrm{C}$ and an air atmosphere at $[\mathrm{M}]=0.60 \mathrm{M}$. 
A

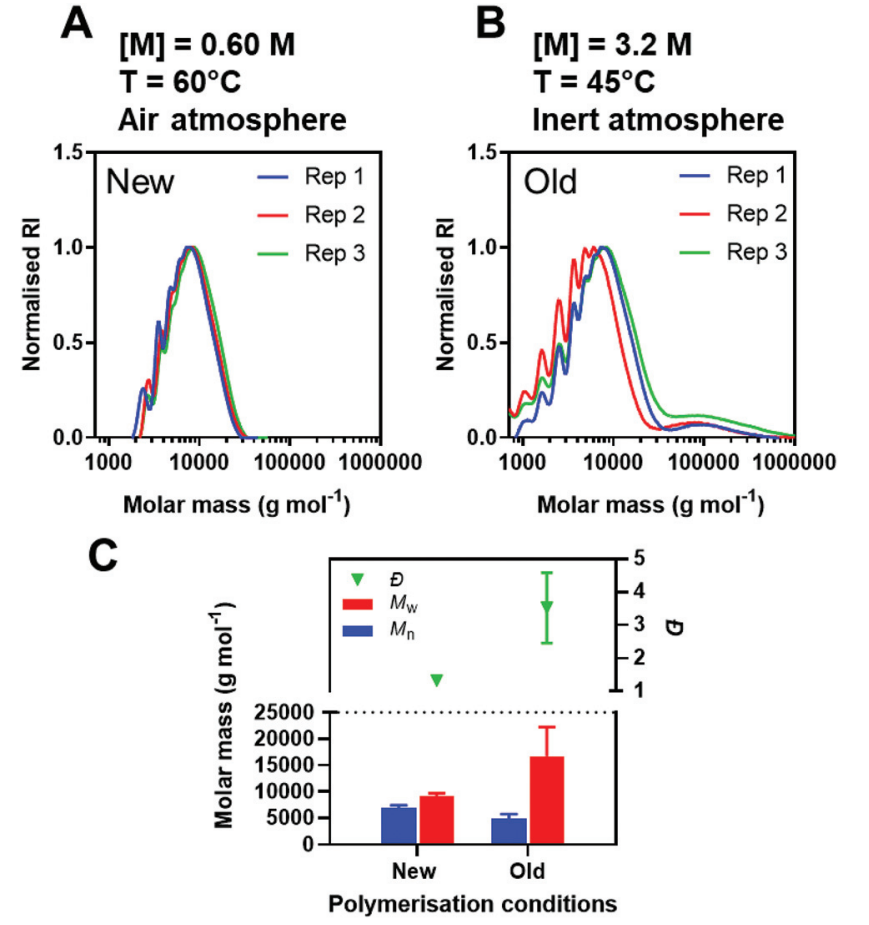

Fig. 5 (A and B) DMF-SEC chromatograms and (C) $M_{w}$ of final polymers after $48 \mathrm{~h}$ reaction between $\mathrm{CBA}$ and $\mathrm{ABOL}$ performed in triplicate. Reactions were performed at $60^{\circ} \mathrm{C},[\mathrm{M}]=0.60 \mathrm{M}$ and an air atmosphere compared to $45^{\circ} \mathrm{C},[\mathrm{M}]=3.2 \mathrm{M}$ and an argon atmosphere.

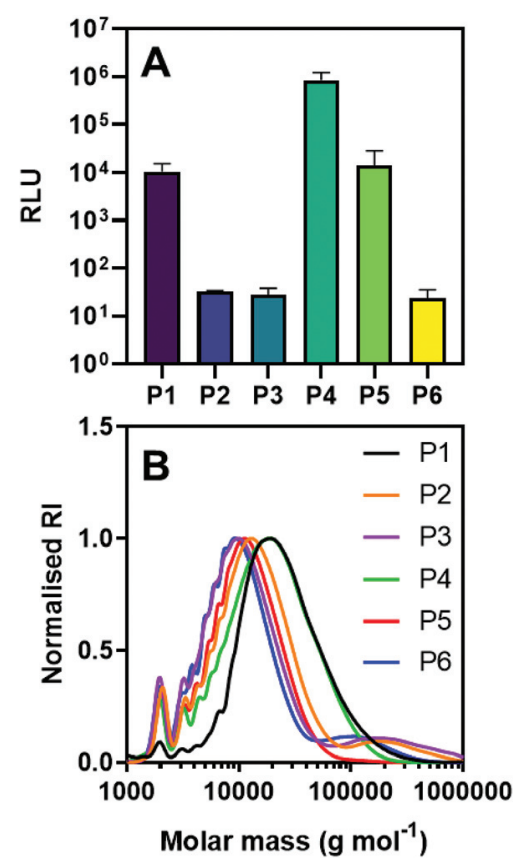

Fig. 6 (A) Transfection of polyplexes with saRNA encoding luciferase at $\mathrm{N} / \mathrm{P}=45$ in HEK $293 \mathrm{~T} .17$ cells $24 \mathrm{~h}$ after treatment. (B) GPC chromatograms of polymers used in transfection studies. different conditions might alter RNA vaccine expression. For these studies we utilised luciferase encoding self-amplifying RNA (saRNA) as a model vaccine. The length of this model saRNA (9382 nt) is in accordance with vaccine constructs e.g. SARS-CoV-2 saRNA is $11551 \mathrm{nt}^{33}$ saRNA constructs have shown great promise in vaccine applications, as their manufacture can be conducted in vitro in a pathogen agnostic manner but can achieve similar efficacy and protective immunity at 1000-fold lower doses than conventional mRNA. ${ }^{34-36}$

There were marked differences in saRNA expression delivered by the various polyplexes derived from the synthesised polymers, dependent on molecular weight distribution (Fig. 6A). Three polymers (P2, P3 and P6) displayed lower luciferase expression $\left(\sim 10^{1} \mathrm{RLU}\right)$, whilst the other three exhibited up to 5 orders of magnitude higher luminescence. Comparing the molecular weight distributions of these two groups (Table S8 $\dagger$ ), it was clear that polymers with bimodal distributions and thus high $D$ severely reduces saRNA transfection. Overall, these results indicate transfection efficiency is significantly enhanced using polymers with monomodal distributions, highlighting the need to minimise radical induced branching as shown in this study. The key question for future development of these materials is therefore what the best compromise between speed and reliability of synthetic method is, while retaining effective transfection in a vaccine setting in vivo.

\section{Conclusions}

In conclusion, we have optimised the $\mathrm{A}_{2}+\mathrm{B}_{2}$ step-growth polymerisation between $\mathrm{CBA}$ and $\mathrm{ABOL}$, yielding conditions that provide low viscosity solutions and minimal side reactions. Our findings suggest that at high concentrations the radical branching side reaction is relatively favourable, with dilution this is partially retarded but is completely inhibited under an air atmosphere. The optimised conditions are now highly reproducible and the timeframe to achieve the $8 \mathrm{~kg} \mathrm{~mol}^{-1}$ target can be accelerated using a microwave synthesiser. These conditions and accelerated timeframe make this synthesis more amenable to scale up, particularly in continuous flow. In terms of the future rapid manufacture of vaccines, the processes to produce these materials are robust, and show small batch-batch variability even with low durations of synthesis. Furthermore, the use of microwaves and simple conditions will facilitate the transfer to flow chemistries, further enhancing the suitability of this method for rapid and scalable manufacture of vaccine excipients. These microwave conditions, utilising standardized equipment, may also provide a reliable method for reproducible decentralized batch synthesis, useful in disease outbreak scenarios where traditional supply chains are constrained. Given the chemical versatility of PAMAM cationic polymer platform, through variation of the two components, we anticipate the findings will extend to synthesis processes with other amines and bisacrylamides. To date we do not yet know the optimal set of physicochemical character- 
istics which produce the best saRNA vaccine properties in vivo, and the correlation between in vitro and in vivo efficacy remains challenging. Nevertheless, the data presented here provide indicators towards more rapid and scalable synthesis of promising nucleic acid vaccine formulation components.

\section{Conflicts of interest}

There are no conflicts of interest to declare.

\section{Acknowledgements}

This research is funded by the Department of Health and Social Care using UK Aid funding and is managed by the Engineering and Physical Sciences Research Council (EPSRC, grant number: EP/R013764/1). The views expressed in this publication are those of the author(s) and not necessarily those of the Department of Health and Social Care. We thank Dr Yunqing Zhu for useful discussions on pABOL synthesis. This research was also funded through the Royal Society through a Wolfson Research Merit Award [WM150086]. We also thank Douglas Crackett and Paul Cooling for expert technical assistance and Carol Turrill for outstanding administrative support.

\section{References}

1 A. C. Anselmo, Y. Gokarn and S. Mitragotri, Nat. Rev. Drug Discovery, 2019, 18, 19-40.

2 R. Kedmi, N. Veiga, S. Ramishetti, M. Goldsmith, D. Rosenblum, N. Dammes, I. Hazan-Halevy, L. Nahary, S. Leviatan-Ben-Arye, M. Harlev, M. Behlke, I. Benhar, J. Lieberman and D. Peer, Nat. Nanotechnol., 2018, 13, 214219.

3 I. Lostale-Seijo and J. Montenegro, Nat. Rev. Chem., 2018, 2, 258-277.

4 K. Muhammad, J. Zhao, I. Ullah, J. T. Guo, X. K. Ren and Y. K. Feng, Biomater. Sci., 2020, 8, 64-83.

5 Q. Cheng, T. Wei, L. Farbiak, L. T. Johnson, S. A. Dilliard and D. J. Siegwart, Nat. Nanotechnol., 2020, 15, 313-320.

6 R. Mout, M. Ray, Y. W. Lee, F. Scaletti and V. M. Rotello, Bioconjugate Chem., 2017, 28, 880-884.

7 J. C. Kaczmarek, P. S. Kowalski and D. G. Anderson, Genome Med., 2017, 9, 60-76.

8 A. K. Blakney, Y. Abdouni, G. Yilmaz, R. Liu, P. F. McKay, C. R. Bouton, R. J. Shattock and C. R. Becer, Biomacromolecules, 2020, 21, 2482-2492.

9 F. Saviano, T. Lovato, A. Russo, G. Russo, C. R. Bouton, R. J. Shattock, C. Alexander, F. Quaglia, A. K. Blakney, P. Gurnani and C. Conte, J. Mater. Chem. B, 2020, 8, 49404949.

10 A. Akinc, D. G. Anderson, D. M. Lynn and R. Langer, Bioconjugate Chem., 2003, 14, 979-988.

11 J. C. Meredith, J. Mater. Chem., 2009, 19, 34-45.
12 R. A. Cordeiro, A. Serra, J. F. J. Coelho and H. Faneca, J. Controlled Release, 2019, 310, 155-187.

13 E. Ben-Akiva, S. E. Witte, R. A. Meyer, K. R. Rhodes and J. J. Green, Biomater. Sci., 2019, 7, 14-30.

14 Y. Rui, D. R. Wilson, J. Choi, M. Varanasi, K. Sanders, J. Karlsson, M. Lim and J. J. Green, Sci. Adv., 2019, 5, eaay3255.

15 S. Z. Duan, D. S. Cao, X. D. Li, H. F. Zhu, M. Lan, Z. Z. Tan, Z. Y. Song, R. Y. Zhu, L. C. Yin and Y. B. Chen, Biomater. Sci., 2020, 8, 290-301.

16 K. L. Kozielski, A. Ruiz-Valls, S. Y. Tzeng, H. GuerreroCazares, Y. Rui, Y. X. Li, H. J. Vaughan, M. GionetGonzales, C. Vantucci, J. Kim, P. Schiapparelli, R. AlKharboosh, A. Quinones-Hinojosa and J. J. Green, Biomaterials, 2019, 209, 79-87.

17 A. Akinc, D. M. Lynn, D. G. Anderson and R. Langer, J. Am. Chem. Soc., 2003, 125, 5316-5323.

18 T. Lovato, V. Taresco, A. Alazzo, C. Sansone, S. Stolnik, C. Alexander and C. Conte, J. Mater. Chem. B, 2018, 6, 6550-6558.

19 A. K. Blakney, Y. Zhu, P. F. McKay, C. R. Bouton, J. Yeow, J. Tang, K. Hu, K. Samnuan, C. L. Grigsby, R. J. Shattock and M. M. Stevens, ACS Nano, 2020, 14, 5711-5727.

20 C. Lin, Z. Zhong, M. C. Lok, X. Jiang, W. E. Hennink, J. Feijen and J. F. J. Engbersen, J. Controlled Release, 2006, 116, 130-137.

21 Z. Zhong, Y. Song, J. F. J. Engbersen, M. C. Lok, W. E. Hennink and J. Feijen, J. Controlled Release, 2005, 109, 317-329.

22 D. M. Lynn, D. G. Anderson, D. Putnam and R. Langer, J. Am. Chem. Soc., 2001, 123, 8155-8156.

23 D. M. Lynn and R. Langer, J. Am. Chem. Soc., 2000, 122, 10761-10768.

24 Y. Liu, D. Wu, Y. Ma, G. Tang, S. Wang, C. He, T. Chung and S. Goh, Chem. Commun., 2003, 2630-2631.

25 C. Lin, C.-J. Blaauboer, M. M. Timoneda, M. C. Lok, M. van Steenbergen, W. E. Hennink, Z. Zhong, J. Feijen and J. F. J. Engbersen, J. Controlled Release, 2008, 126, 166-174.

26 P. Gurnani, A. K. Blakney, R. Terracciano, J. E. Petch, A. J. Blok, C. R. Bouton, P. F. McKay, R. J. Shattock and C. Alexander, Biomacromolecules, 2020, 21, 3242-3253.

27 A. Goto and T. Fukuda, Prog. Polym. Sci., 2004, 29, 329-385.

28 J. Yeow, R. Chapman, A. J. Gormley and C. Boyer, Chem. Soc. Rev., 2018, 47, 4357-4387.

29 M. Nüchter, B. Ondruschka, W. Bonrath and A. Gum, Green Chem., 2004, 6, 128-141.

30 K. Kempe, C. R. Becer and U. S. Schubert, Macromolecules, 2011, 44, 5825-5842.

31 T. N. Glasnov and C. O. Kappe, Macromol. Rapid Commun., 2007, 28, 395-410.

32 J. K. Stille, J. Chem. Educ., 1981, 58, 862.

33 P. F. McKay, K. Hu, A. K. Blakney, K. Samnuan, J. C. Brown, R. Penn, J. Zhou, C. R. Bouton, P. Rogers, K. Polra, P. J. C. Lin, C. Barbosa, Y. K. Tam, W. S. Barclay and R. J. Shattock, Nat. Commun., 2020, 11, 3523. 
34 C. Zhang, G. Maruggi, H. Shan and J. Li, Front. Immunol., 2019, 10, 594.

35 A. B. Vogel, L. Lambert, E. Kinnear, D. Busse, S. Erbar, K. C. Reuter, L. Wicke, M. Perkovic, T. Beissert, H. Haas, S. T. Reece, U. Sahin and J. S. Tregoning, Mol. Ther., 2018, 26, 446-455.
36 A. J. Geall, A. Verma, G. R. Otten, C. A. Shaw, A. Hekele, K. Banerjee, Y. Cu, C. W. Beard, L. A. Brito, T. Krucker, D. T. O'Hagan, M. Singh, P. W. Mason, N. M. Valiante, P. R. Dormitzer, S. W. Barnett, R. Rappuoli, J. B. Ulmer and C. W. Mandl, Proc. Natl. Acad. Sci. U. S. A., 2012, 109, 14604-14609. 